\title{
Numerical simulation of superheated jets using an Eulerian method
}

\author{
Konstantinos Lyras ${ }^{1}$, Siaka Dembele ${ }^{1}$, C. Madhav Rao Vendra ${ }^{2}$, Jennifer Wen ${ }^{2 *}$ \\ ${ }^{1}$ Department of Mechanical and Automotive engineering, Kingston University London, United \\ Kingdom \\ ${ }^{2}$ School of Engineering, University of Warwick, Coventry, United Kingdom \\ *Corresponding author: jennifer.wen@warwick.ac.uk
}

\begin{abstract}
Flash boiling is the rapid phase change of a pressurised fluid that emerges in ambient conditions below its vapour pressure. Flashing can occur either inside or outside the nozzle depending on the local pressure and geometry and the bubble formation leads to interfacial interactions that eventually influence the emerging spray. Lagrangian methods which exist in literature to simulate the flash atomisation and inter-phase heat transfer employ many simplifying assumptions. Typically, sub-models used for the break-up, collisions and evaporation introduce an extensive empiricism that might result in unrealistic predictions for cases like flashing. In this study, a fully Eulerian approach is selected employing the $\Sigma-Y$ model proposed by Vallet and Borghi. The model tracks liquid structures of any shape and computes the spray characteristics comprising a modified version for the transport equation of the surface density. The main goal of this study is to investigate the performance of this model in flash boiling liquids using the Homogeneous Relaxation Model (HRM) developed by Downar-Zapolski, a model capable of capturing the heat transfer under sudden depressurisation conditions accounting for the non-equilibrium vapour generation. The model in this present study considers that the instantaneous quality would relax to the equilibrium value over a given timescale which is calculated using the flow field values. A segregated approach linking the HRM and $\Sigma-Y$ is implemented in a compressible formulation in an attempt to quantify the effects of flash boiling in the spray dynamics. The developed model is naturally implemented in RANS in a dedicated solver HRMSonicELSAFoam. Results from simulations of two-phase jets of different subcooled fluids through sharp-edged orifices show that the proposed approach can accurately simulate the primary atomisation and give reliable predictions for the droplet sizes and distribution. Strong effects of the flashing and turbulent mixing on the jet are demonstrated. The model is tested for turbulent flows within small nozzles and was developed within the open source code OpenFOAM.
\end{abstract}

\section{Keywords}

Flash-boiling, atomisation, ELSA model.

\section{Introduction}

Flashing jets occur when a high-pressure liquid flowing through a nozzle or an orifice is suddenly exposed to a low-pressure environment, becoming superheated if it is not already so. Flashing is characterised by a rapid phase change along the jet and bubble nucleation within the liquid core that influences the spray formation [1, 2]. Flashing is very important in safety studies in cases of accidental releases of a liquefied flammable gas through a small crack in the pipeline system. In the aerosol industry it can be used to control nucleation having the advantage of producing sprays with very fine droplets within small domains [3]. In its dense part, the two-phase jet might appear in different forms like bubbly, slug or annular and the nucleation is possible to start upstream of the orifice (a process generally termed internal flashing) or at some distance downstream of the orifice (external flashing). The geometry and the nozzle length-to-diameter ratio, the degree of superheat or subcooling, the storage and ambient conditions play a crucial role in the atomisation process. Flashing occurs either if a liquid follows an isothermal depressurisation or an isobaric heating. In both cases, the fluid fails to adjust to the local changes in pressure and temperature admitting a metastable state which makes the process more challenging to understand.

Lagrangian modelling approaches usually rely on submodels for flash vaporisation (such as in [4]) and incorporate them in the concept of liquid parcels. The idea of parcels splits the modelling of the rapid boiling and aerodynamic fragmentation of the jet employing single-droplets models. Despite the simplifications it suffers from, it is a logical approach to the problem since after the primary atomisation and the consequent break-up, the liquid structures become small enough to resemble spherical droplets. Experimental studies for water [5-7] and various cryogenic fluids like liquid nitrogen in [8] indicate that two-phase jets emerging through a nozzle might begin flashing with a severe impact on the spray. Numerical works of Lee et al. in [9] and Schmidt et al. in [10] also agree with this change in the disintegration regime. In spite of its significance, detailed numerical studies of flash boiling atomisation are scarce. Here, a novel approach is proposed to simulate the nozzle flow and the primary atomisation in an Eulerian framework. The method tracks the interface of the spray in a compressible conservative manner using the volume of fluid (VOF) method and is combined with the HRM implementation of Schmidt et al. [10]. The method switches to a Lagrangian behaviour for the smaller liquid structures employing the $\Sigma-Y$ model of Vallet and Borghi [11]. The simulations considered here concern R134a jets which is an example a cryogenic liquid with strong vaporisation effects when released in the atmosphere. For this reason, a modified formulation is suggested for the $\Sigma-Y$ model, adjusting the surface density equation to accommodate simulations of superheated jets. 


\section{Mathematical formulations and methods}

The Eulerian-Lagrangian Spray Atomisation (ELSA) model was established after the first formulation of $\Sigma-Y$ model in [11-12]. In ELSA, the two-phase flow consists of the liquid and gas, which vary in density and the flow is studied as one single phase, solving one equation for mass and momentum which are omitted here for the sake of brevity. The same stands for the VOF method. The liquid mass fraction $Y_{l}$ is used as a marker function to track the liquid. The liquid mass fraction is calculated by the following equation,

$$
\frac{\partial \bar{\rho} \tilde{Y}_{l}}{\partial t}+\frac{\partial \bar{\rho} \tilde{Y}_{l} \tilde{u}_{j}}{\partial x_{j}}=-\frac{\partial \bar{\rho} R_{j l}}{\partial x_{j}}
$$

where $R_{j l}$ is the turbulent liquid flux given by,

$$
R_{j l}=-\frac{\nu_{t}}{S c_{t}} \frac{\partial \tilde{Y}_{l}}{\partial x_{j}}
$$

where $u_{j}, \rho, \nu_{t}, S c_{t}$ are the common velocity of the liquid and gas in the $j$ direction, the mixture density, the kinematic turbulent viscosity and the turbulent Schmidt number respectively. Here only RANS approach is treated and $\tilde{\phi}$ denotes the mass weighted Favre average of a variable $\phi$ decomposed into a mean part $\bar{\phi}$ and a fluctuating part $\phi^{\prime \prime}$. The classical Navier-Stokes equations are solved in a fully Eulerian context using VOF and the flow variables such as density, viscosity are averaged using the vapour mass fraction $x$, which is calculated with the following transport equation,

$$
\frac{\partial \bar{\rho} \tilde{x}}{\partial t}+\frac{\partial \bar{\rho} \tilde{u_{j}} \tilde{x}}{\partial x_{j}}=\Gamma
$$

Here $\Gamma$ is the source term that stands for the interfacial mass transfer and is related to the vapour mass production rate. This term is calculated using the HRM developed by Downar Zapolski in [13] and is written as a first order approximation as,

$$
\Gamma=\rho \frac{D x}{D t}=\rho \frac{x^{*}-x}{\Theta}
$$

Here $x^{*}$ is the equilibrium value of $x$ and $\Theta$ is a timescale in which $x$ would relax to $x^{*}$. The equilibrium value $x^{*}$ is calculated here assuming isenthalpic conditions as,

$$
x^{*}=\frac{h-h_{l, s a t}}{h_{v, s a t}-h_{l, s a t}}
$$

In this formulation, $h_{l, s a t}, h_{v, s a t}$ are the saturated enthalpies of liquid and vapour state. The timescale for the model is calculated as,

$$
\Theta=\Theta_{0} \alpha^{-0.257} \psi^{-2.24}
$$

and is validated for flashing releases of water at pressures up to ten bar. The non-dimensional pressure $\psi$ is equal $\left(p_{\text {sat }}-p\right) / p_{\text {sat }}$. The timescale $\Theta$ is a function of the constant $\Theta_{0}=6.51 \times 10^{-4}[s]$ and the void fraction given by,

$$
\alpha=\frac{\rho_{l}-\rho}{\rho_{l}-\rho_{v}}
$$

where $\rho_{l}$ and $\rho_{v}$ are the liquid and vapour densities. The ideal gas law is used for $\rho_{v}$ and $\rho_{l}$ is calculated using compressibility and a reference state as,

$$
\rho_{l}=\rho_{r e f}+\frac{\partial \rho}{\partial p}\left(p-p_{r e f}\right)
$$

\section{The Eulerian-Lagrangian Spray Atomisation (ELSA) model}

The ELSA model is used to model the atomisation process and has been extensively tested for spray injection in the automotive industry. It is used here to predict the characteristics of the liquid structures in the dense and dilute parts of the spray. The innovative idea that is introduced in ELSA, is the mean liquid/gas interface density $\Sigma$ for describing the spray. This definition is not limited to the assumption of spherical droplets which is a standard in many Lagrangian methods in commercial and open-source software. The interface density can be considered as the amount of spatial surface of liquid per unit volume at a given position, hence $\Sigma$ has units of inverse length, $m^{-1}$. In finite volume methods, the volume is the control volume as shown in figure 1. 


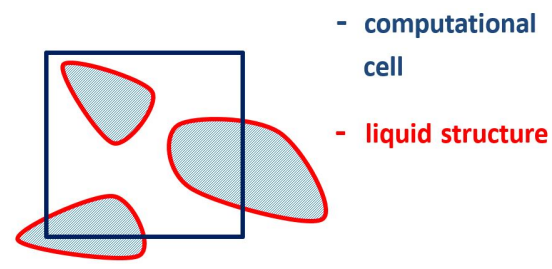

Figure 1. Surface density at a control volume. Bold red colour inside the computational cell indicates the liquid surface and the bold blue line is the control volume in a 2D projection.

The ELSA model has been originally developed and validated in RANS and is proven to accurately model the turbulent mixing $[15,20]$. It has been modified since its first appearance. Here we use the formulation proposed by Menard et al. [14]. The model was modified by Lebas et al. [15] and the $\Sigma$ equation can be written as,

$$
\frac{\partial \bar{\Sigma}}{\partial t}+\frac{\partial \tilde{u}_{j} \bar{\Sigma}}{\partial x_{j}}=\frac{\partial}{\partial x_{j}}\left(\frac{\nu_{t}}{S c_{t}} \frac{\partial \bar{\Sigma}}{\partial x_{j}}\right)+\Psi\left(S_{\text {init }}+S_{\text {turb }}\right)+(1-\Psi)\left(S_{\text {coll }}+S_{2 n d B U}\right)+S_{v a p}
$$

The model consists of several source terms on the RHS, which are associated to different processes that might have impact on the atomisation. The source terms can be calculated via different approaches. Here they are split for the dense and dilute part of the spray using an indicator function $\Psi$ which is equal to one if the liquid mass fraction, $\tilde{Y}_{l}$ is between 1 and 0.5 and is zero for cells with liquid mass fraction less than 0.1 . The indicator function can be written as a function of the liquid volume fraction, $\phi_{l}$, where $\phi_{l}=\bar{\rho} \tilde{Y}_{l} / \bar{\rho}_{l}$ as,

$$
\Psi\left(\phi_{l}\right)=H\left(\phi_{l}-0.1\right) H\left(\phi_{l}-0.5\right)+\left(H\left(\phi_{l}-0.1\right)-H\left(\phi_{l}-0.5\right)\right)\left(2.5 \phi_{l}-0.25\right)
$$

where $H()$ is the Heaviside step function. The term $S_{\text {init }}$ corresponds to the minimum liquid/gas surface produced in the atomisation process and is larger where the gradient of liquid mass fraction is higher according to,

$$
S_{\text {init }}=2 \frac{\mu_{t}}{S c_{t}} \frac{6 \bar{\rho}}{\rho_{l} \rho_{g} l_{t}} \frac{\partial \tilde{Y}_{l}}{\partial x_{i}} \frac{\partial \tilde{Y}_{l}}{\partial x_{i}}
$$

This expression is used in cases where $\tilde{Y}_{l}\left(1-\tilde{Y}_{l}\right)<0.001$. Otherwise the second fraction in equation 11 is equal to $\frac{\bar{\Sigma}}{\tilde{Y}_{l}\left(1-\tilde{Y}_{l}\right)}$. In the previous equation, $\rho_{g}$ is the density of ambient gas (still air in the present study) and $l_{t}$ is the turbulent length scale. Since the $S S T k-\omega$ model is used here, it is written as,

$$
l_{t}=C_{\mu}^{3 / 4} \frac{k^{1 / 2}}{\omega}
$$

where $C_{\mu}$ is a constant equal to 0.09. $S_{\text {turb }}$ is the term responsible for the production or destruction of the interface density due to stretching caused by turbulence and collisions/coalescences in the dense part of the spray. It is assumed that interface will be created or destroyed due to turbulence until $\Sigma$ reaches an equilibrium value, $\Sigma_{\text {turb }}^{*}$. The turbulent time-scale $\tau_{t}$ for this process is calculated from the turbulence model as,

$$
\tau_{t}=\frac{1}{\omega}
$$

Considering a Weber number equal to one at the equilibrium, the source term is then calculated as,

$$
S_{\text {turb }}=\frac{\bar{\Sigma}}{\tau_{t}}\left(1-\frac{\bar{\Sigma}}{\Sigma_{\text {turb }}^{*}}\right)
$$

At the dilute part of the spray, the liquid structures are treated in a Lagrangian manner. The source term for the surface creation/destruction due to collisions in the dilute areas is written in a similar way as in equation 14 . The collision time-scale $\tau_{\text {coll }}$ is calculated as,

$$
\tau_{\text {coll }}=\frac{1}{\bar{\Sigma} \sqrt{\frac{2 \tilde{k}}{3}}}
$$

where $\tilde{k}$ is the turbulent kinetic energy. Hence, the collisions source term is equal to,

$$
S_{\text {coll }}=\frac{\bar{\Sigma}}{\tau_{\text {coll }}}\left(1-\frac{\bar{\Sigma}}{\Sigma_{\text {coll }}^{*}}\right)
$$


The additional equilibrium term $\Sigma_{\text {coll }}^{*}$ is calculated via an equilibrium Weber number,

$$
W e_{\text {coll }}^{*}=\frac{\rho_{l} u_{\text {coll }}^{2} D_{32}}{\sigma_{l}}
$$

Where $\sigma_{l}$ is the surface tension of liquid. The characteristic collision velocity $u_{\text {coll }}$ is calculated via,

$$
u_{\text {coll }}=\sqrt{\frac{2}{3} \tilde{k}}
$$

The diameter $D_{32}$ is the characteristic size of the droplet, equivalent to the Sauter mean diameter and equals to,

$$
D_{32}=\frac{6 \bar{\rho} \tilde{Y}_{l}}{\rho_{l} \bar{\Sigma}}
$$

The equilibrium Weber $W e_{\text {coll }}^{*}$ is taken equal to 12 and the $\Sigma_{\text {coll }}^{*}$ is now calculated for equation 16 . The next term to model in equation 9 is $S_{2 n d B U}$, which comprises the surface production due to secondary break-up and is updated using an expression like equation 16. The time-scale $\tau_{2 n d B U}$ of the break-up is calculated using the experimental work of Pilch and Erdman [16]. This is a characteristic time where the liquid droplet will break-up due to RayleighTaylor or Kelvin-Helmholtz instabilities and is given by,

$$
\tau_{2 n d B U}=T \frac{D_{32}}{u_{r e l}} \sqrt{\frac{\rho_{l}}{\rho_{g}}}
$$

where the relative velocity between the two phases, $u_{r e l}$ can be computed using equation 2 as,

$$
u_{r e l}=\frac{R_{j l}}{\tilde{Y}_{l}\left(1-\tilde{Y}_{l}\right)}
$$

$T$ is the total dimensionless time defined as the time when the drop and its fragments no longer break-up and is taken from [16]. The equilibrium Weber number for the secondary break-up is equal to,

$$
W e_{2 n d B U}^{*}=12\left(1+1.077 O h^{1.6}\right)
$$

and assuming the effect of viscosity negligible, an equilibrium $\Sigma_{2 n d B U}^{*}$ is obtained and $S_{2 n d B U}$ is updated. Finally, the last term in equation 9 is $S_{v a p}$, which is responsible for the change in interface density due to evaporation. This term is usually omitted in the literature since there is no available model valid for all the spray regions. In superheated liquids such as Liquefied Natural Gas (LNG) or in many refrigerants, evaporation could be important in the equation for $\Sigma$. A simple correlation is introduced here,

$$
S_{\text {vap }}=-\frac{\bar{\Sigma}}{\Theta}\left(\frac{x^{*} \rho^{*}}{\rho}-x\right)
$$

where the "*" symbol stands for the thermodynamic equilibrium of each illustrated variable. The equations presented are solved in a segregated approach using the finite volume method. The method in this study was developed using OpenFOAM [17]. All the variables are stored in the centre of the control volumes and the interpolated fluxes are stored at the centre of the cell faces. The PIMPLE algorithm is used for the pressure velocity coupling. The algorithm proposed by Schmidt et al. in [10] is used inserting the contribution of phase change into the pressure equation. The PIMPLE algorithm is coupled with HRM in a way that considers the non-equilibrium vapour generation in the pressure-velocity coupling which is absent in standard OpenFOAM. Additionally, the available models for modelling the droplet evaporation were found to give less accurate predictions in the vapour mass fraction and velocity, especially in the dense spray region, something that is not observed with HRM.

\section{Results and discussion}

Numerical simulations were performed using the series of experiments from the FLIE project [18-19] investigating flashing releases of R134A (1,1,1,2 - Tetrafluoroethane: CF3-CH2F). The experimental domain consists of a high pressure region where R134A is stored at a pressure above its vapour pressure at ambient conditions (663 $k P a$ at $293.15 \mathrm{~K}$ ). The liquid passes through a nozzle of diameter $D$ and length $L$ and is released into a low-pressure region which is equal to the atmospheric pressure. The computational domain and its discretisation consisting of 2.0 million hexahedral cells are shown in figure 2. The curvature at the walls in the nozzle exit is not considered for the present simulations. The liquid core is more dense around the centreline of the jet, which indicates that finer mesh is needed there. The physical parameters for the simulations are shown in Table 1. The cases tested here concern the geometry in which $L=4 \mathrm{~mm}$ and $D=2 \mathrm{~mm}$. Keeping in mind the importance of the flash boiling or the geometry impact on the spray, the flow is simulated inside the nozzle avoiding arbitrary assumptions for the jet inlet in the lower pressure region. Figure 3 illustrates the velocity distribution. The jet is emerging in the atmosphere with a velocity of approximately $32 \mathrm{~m} / \mathrm{s}$, which is in good agreement with the experimental observations. The liquid core that is observed in the vicinity of the centreline of the jet, has the highest velocity. 
Table 1. Physical properties for simulations.

\begin{tabular}{c|c}
\hline & Physical parameters for simulations \\
\hline Inlet pressure & $800 \mathrm{kPa}$ \\
Inlet temperature & $293.35 \mathrm{~K}$ \\
Outlet pressure & $100 \mathrm{kPa}$ \\
Outlet temperature & $298 \mathrm{~K}$ \\
$L / D$ & 2 \\
Superheat degree & $47 \mathrm{~K}$
\end{tabular}

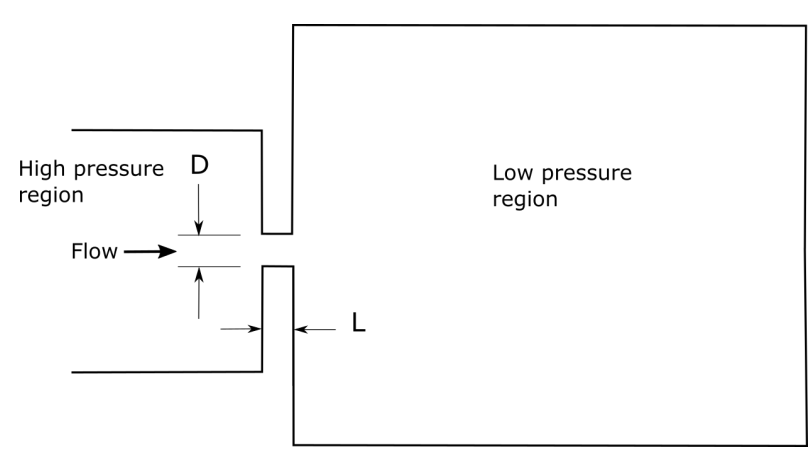

(a) Simplified sketch of the domain for the experiments in [19].

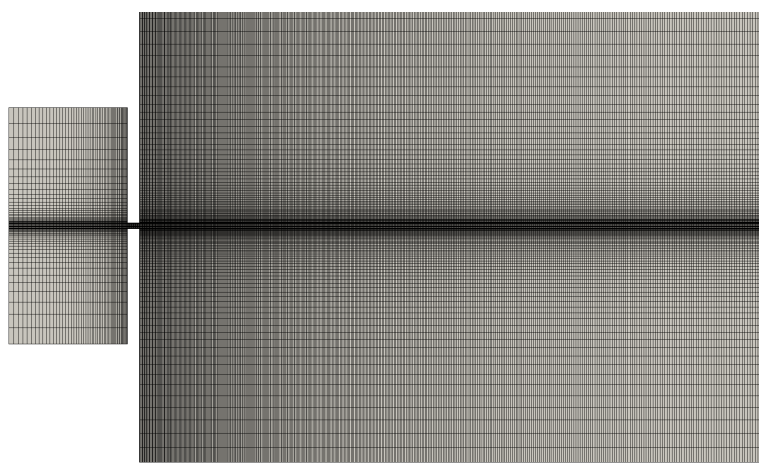

(b) Discretisation of the domain.

Figure 2. Representation of the domain

This is obvious in both inside and outside the nozzle. As long as we move along the centreline the jet morphology changes with an enhanced evaporation on its surface. The change in the jet regime at the beginning of the release is clearer in figure 4.

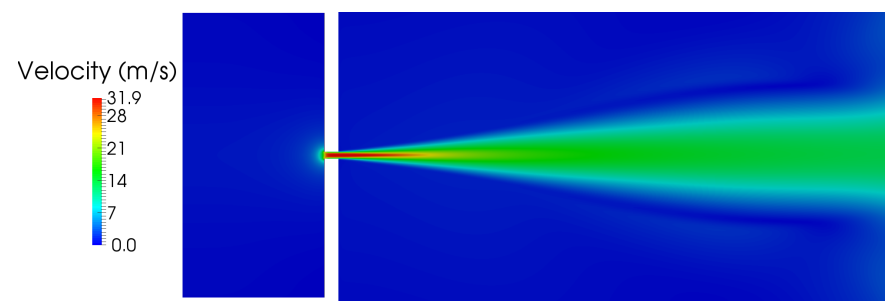

(a) Velocity profile projection for the selected case.

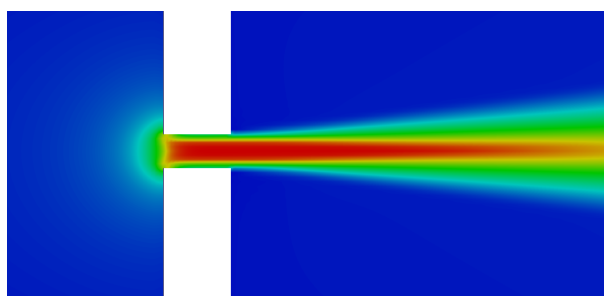

(b) A closer caption of the velocity.

Figure 3. Converged jet velocity.

The liquid core is dense with a high number of liquid ligaments close to the nozzle and the liquid volume fraction has its highest value, close to one. A few diameters away from the nozzle exit, the liquid volume fraction decreases until it reaches the lowest limit of zero. For a distance up to $200 \mathrm{D}$, two regions of this decrease can be identified which correspond to different slopes in the graph in figure 4-b. The same trends are observed in other numerical works (see in [20]). The bandwidth of these regions is affected by the thermophysical properties and is expected to differ for cryogenic releases and non-evaporating jets. Previous studies in flashing jets indicate a rapid phase change as soon as the local pressure drops lower than the saturation pressure. The initiation of this phase change is more likely to happen within the nozzle for longer nozzles but is also possible for short nozzles with $L / D$ less than seven like here, even in low superheat degrees [8]. The jet vaporises within the nozzle, and the flow separation at the sharp inlet corners results in a pressure drop and consequently in phase change (for more details see in [21]). Because of this, the flow becomes two-phase and the liquid volume fraction decreases close to the nozzle walls as shown in figure 4-a. The same behaviour is observed in the density. Hence, the jet at the nozzle exit, cannot be considered as a pure liquid. Additionally, the method presented in the previous chapter, can predict the amount of the vaporised liquid giving a realistic prediction for the jet morphology downstream.

Another important parameter in the atomisation modelling is the mean interface density and its evolution. $\Sigma$ is calculated via equation 9 comprising all the possible processes that could cause an increase or decrease in the liquid/gas interface. For this reason a second order of accuracy bounded scheme for the convective terms in the $\Sigma$-equation is used. A typical distribution of $\Sigma$ is illustrated in figure 5 . It is expected to be higher closer to the nozzle exit where the initiation term Sinit dominates. The source term $S_{\text {init }}$ dominates the $\Sigma$ generation initially and together with $S_{\text {turb }}$ are the most important source terms in the primary atomisation region. On the contrary the 


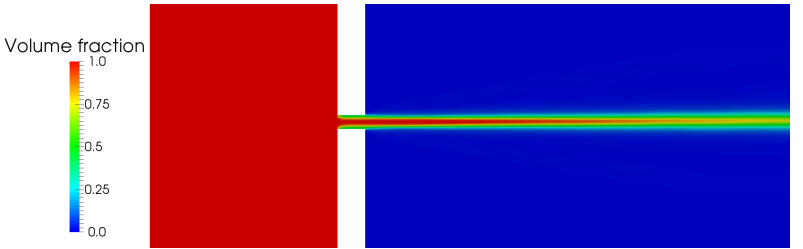

(a) In the whole domain.

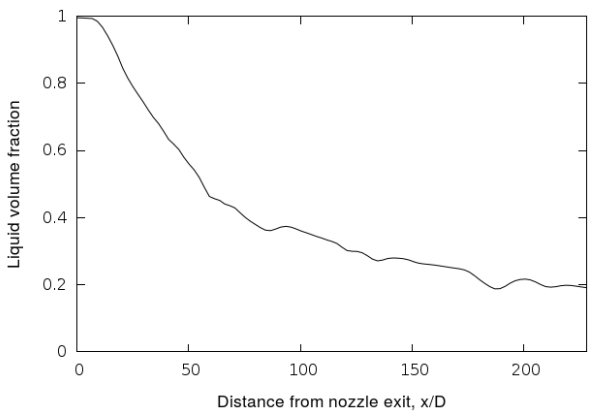

(b) In the centreline of the jet.

Figure 4. Liquid volume fraction for the properties of Table 1.

terms $S_{\text {coll }}, S_{2 n d B U}$ are less important in the vicinity of the nozzle exit hence $\Psi$ is equal to 1 in this case. The terms $S_{\text {coll }}, S_{2 n d B U}$ are dominant in the secondary break-up region where droplets are generated due to aerodynamic break-up and collisions. These terms prevail in the $\Sigma$-equation and $\Psi$ tends to zero for the diluted regions. The terms $S_{\text {init }}$ and $S_{\text {turb }}$ are expected to be of minor importance in this case. The source term $S_{v a p}$ is more important in the primary atomisation and two/three dimensional numerical investigations showed that usually is smaller than the other source terms in equation 9. Definitely, further examination of equation 23 must be conducted. From equation 11, the initial interface density is a function of the liquid mass fraction gradient which is greater close to the injection region. After the injection, the jet starts to fragment and interface is created. Consequently, $\Sigma$ is increasing. During the primary atomisation, large blobs and ligaments form, which is also reported in [19] in the experiments. After reaching a peak value, $\Sigma$ starts to decrease rapidly. Some diameters after the peak value, the interface density reduces to $\mathcal{O}(10)$. In this region, apart from the evaporation process, the secondary break-up takes place and causes the large ligaments to burst into smaller droplets which leads to an increase in the interface density. That is why the source term $S_{2 n d B U}$ is positive in equation 9 . The contribution of the source term due to droplet collisions, $S_{\text {coll }}$ is also included in this part of the spray. The droplet size is defined in the regions where the liquid mass fraction and interface density are non-zero. It is calculated via equation 19 and some two-dimensional results are shown in figure 5 for the Sauter mean diameter $\left(D_{32}\right)$ along the jet centreline. The $D_{32}$ is higher close to the nozzle exit where the jet is almost pure liquid at the centreline. After almost 5 diameters, the $D_{32}$ order is of some hundreds of microns. As mentioned before, flashing is a process very efficient in the aerosol industry for producing fine sprays. Hence, the resulting $D_{32}$ values are reasonable and in relatively good agreement with the experimental data from [19]. Three-dimensional simulations performed showed that along the radial axis, $D_{32}$ is maximum at the jet centreline and decreases along the radial axis.

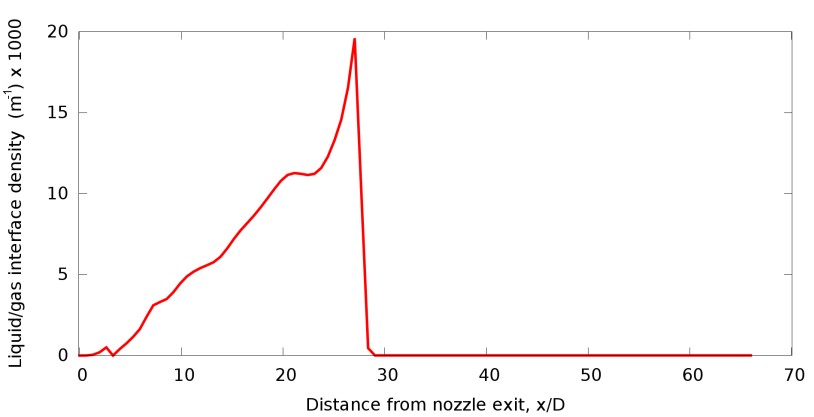

(a) Axial interface density distribution.

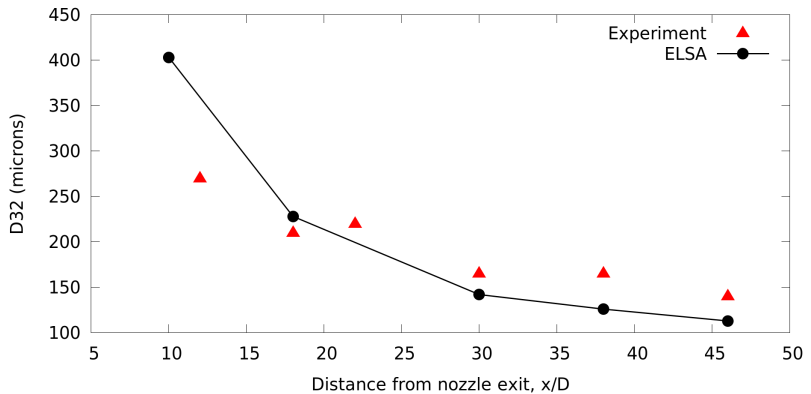

(b) Sauter mean diameter in the centreline of the jet.

Figure 5. ELSA model simulations.

The axial velocity obtained from ELSA at the primary atomisation region is shown in figure 6 compared to the PIV and PDA experimental results. The velocity ranged in the simulations between $32 \mathrm{~m} / \mathrm{s}$ in the dense region, and $1 \mathrm{~m} / \mathrm{s}$ in the diluted regions. In the simulations, the peak velocity is observed a few diameters after the jet exits to the atmosphere whereas in the experiments the peak velocity is reported in the region between $10-18 D$ as illustrated in figure 6 . The velocity obtained from ELSA model is high and almost constant within this range, but decreases with further increasing the distance from the nozzle exit. For example it gets half at $x / D=110$ [19].

\section{Conclusions}

An Eulerian-Lagrangian Atomisation Spray method using RANS, implemented within OpenFOAM, is presented. The model follows the original implementation of $\Sigma-Y$ model, solving two equations, one for the liquid mass fraction, and another one for the interface density. Source terms for accounting for the creation/destruction of the interface due to mechanical and thermodynamic effects are used in the modelling. A novel expression for the change in sur- 


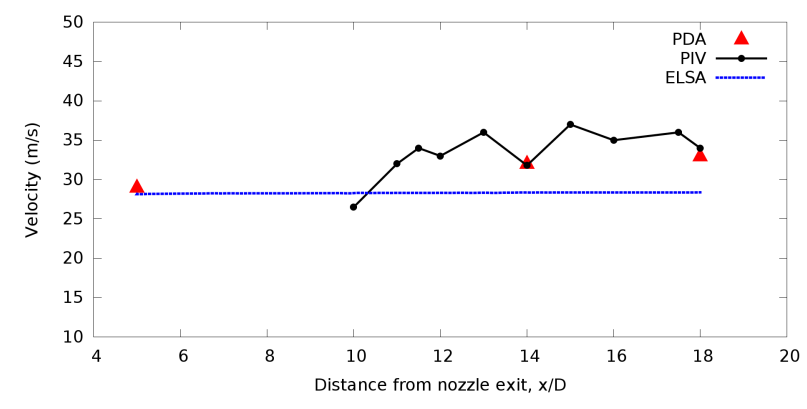

Figure 6. Velocity along the jet centreline.

face density in cryogenic jets is used here and is inserted in the $\Sigma$-equation. The applications of the method include fuel atomisers, accidental releases and other situations of a sudden pressure drop of a superheated/sub-cooled liquids in a low pressure environment.

The ELSA approach, combines the advantages of Eulerian and Lagrangian frameworks, and uses explicit formulations for modelling the droplet characteristics like the Sauter mean diameter. Preliminary results show that the method can properly describe the droplet sizes along the jet centreline. Further research should be made for the implicit/explicit treatment of the source terms in the $\Sigma-Y$ model and the impact of the turbulence modelling approach on the primary atomisation.

\section{Acknowledgements}

The authors gratefully thank the financial support by the Marie Curie Action of the 7th framework Programme of the European Commission. The current work is part of the SafeLNG project (project ID: 606754) on the numerical characterisation and simulation of the complex physics underpinning the safe handling of liquefied natural gas (2014-2018).

\section{Nomenclature}

$C_{\mu} \quad$ constant of turbulence model [-]

$D \quad$ diameter $[m]$

$D_{32} \quad$ Sauter mean diameter, SMD $[m]$

$h \quad$ enthalpy $[\mathrm{J} / \mathrm{kg}]$

$H \quad$ Heaviside step function [-]

$k \quad$ kinetic energy $\left[\mathrm{m}^{2} \mathrm{~s}^{-2}\right]$

$L \quad$ length of nozzle $[\mathrm{m}]$

$l_{t} \quad$ turbulent length scale $[\mathrm{m}]$

Oh Ohnesorge number, $\mu_{l} / \rho_{l} D_{32} \sigma_{l}[-]$

$p \quad$ pressure $[k P a]$

$S_{\text {init }} \quad$ minimum liquid/gas interface production $\left[\mathrm{m}^{-1} \mathrm{~s}^{-1}\right.$ ]

$S_{\text {turb }} \quad$ source term for interface production/destruction due to turbulence $\left[\mathrm{m}^{-1} \mathrm{~s}^{-1}\right]$

$S_{\text {coll }} \quad$ interface density source term due to collisions $\left[\mathrm{m}^{-1} \mathrm{~s}^{-1}\right]$

$S_{2 n d B U}$ interface density source term due to secondary break-up $\left[m^{-1} s^{-1}\right]$

$S_{v a p} \quad$ interface density source term due to evaporation $\left[\mathrm{m}^{-1} \mathrm{~s}^{-1}\right]$

$S c_{t} \quad$ turbulent Schmidt number, $\mu / \rho D_{m}[-]$, where $D_{m}=$ mass diffusivity $\left[m^{2} s^{-1}\right]$

$t \quad$ time $[s]$

$u_{j} \quad$ velocity $[\mathrm{m} / \mathrm{s}]$

$u_{\text {coll }} \quad$ characteristic collision velocity $[\mathrm{m} / \mathrm{s}]$

$W e_{\text {coll }}^{*}$ equilibrium Weber number characteristic for droplets collisions $[\mathrm{m} / \mathrm{s}]$

$W e_{2 n d B L}^{*}$ equilibrium Weber number characteristic for secondary break-up $[\mathrm{m} / \mathrm{s}]$

$x \quad$ vapour mass fraction [-]

$x^{*} \quad$ equilibrium vapour mass fraction [-]

$\phi_{l}, \tilde{Y} \quad$ liquid volume fraction, mass weighted Favre averaged mass fraction [-]

$\alpha \quad$ void fraction [-]

$\Gamma \quad$ interfacial mass transfer in the HRM $\left[\mathrm{kgm}^{-3} \mathrm{~s}^{-1}\right]$

$\Theta \quad$ relaxation time scale $[s]$

$\Theta_{0} \quad$ constant in the HRM $[s]$

$\mu, \mu_{t} \quad$ dynamic viscosity, dynamic turbulent viscosity $\left[\mathrm{kgm}^{-1} \mathrm{~s}^{-1}\right]$

$\nu_{t} \quad$ kinematic eddy viscosity $\left[\mathrm{m}^{2} / \mathrm{s}\right]$

$\rho \quad$ density $\left[\mathrm{kgm}^{-3}\right]$

$\sigma \quad$ surface tension $\left[\mathrm{kgs}^{-2}\right]$ 


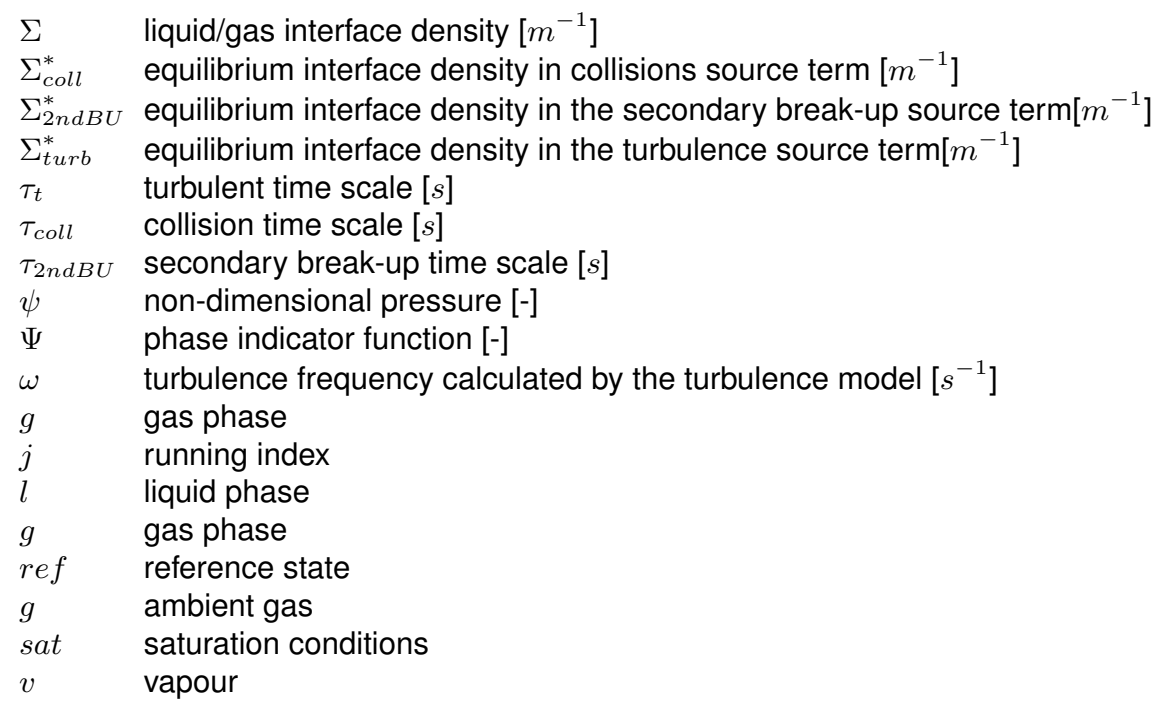

\section{References}

[1] Oza, R.D.,Sinnamon, J.F., 1983, "An experimental and analytical study of flash boiling fuel injection”, SAE paper, 830590.

[2] Witlox, H., Bowen, P., 2002, "Flashing liquid jets and two-phase dispersion. A review". Contract research report 403/2002, HSE.

[3] Sher, E.,Bar-Kohany, T., Rashkovan A. 2008, "Flash boiling atomization", Progress in Energy and Combustion Science, 34 (4), pp 417-439.

[4] Zuo B., Gomes A.M., Rutland C.J., 2000, "Modelling Superheated Fuel Spray Vaporization”, Int. Journal of Engine Research, (1), pp. 321-326.

[5] Reitz R., 1990, "A Photographic Study of Flash-Boiling Atomization", Aerosol Science and Technology, (12) 3, pp. 561-569.

[6] Park C.K., Park J. W., Chung M. K., Chun M. K., 1997, "An empirical correlation for Critical Flow Rates of Subcooled Water Through Short Pipes with Small diameters", Journal of the Korean Nuclear society, (29) 1, pp. 35-44.

[7] Xu J., Chin T., Yang L., 1995, "Two-Phase Critical Discharge of Initially Saturated or Subcooled Water Flowing in Sharp-Edged Tubes at High Pressure”, Journal of Thermal Science, (4) 3, pp. 193-199.

[8] Simoneau R.,1975, "Maximum two-phase flow rates of subcooled Nitrogen through a sharp-edged orifice", NASA TM X-71760.

[9] Lee J., Madabhushi R., Fotache C., Gopalakrishnan S., Schmidt D., 2009, "Flashing flow of superheated jet fuel", Proceedings of the Combustion Institute, (31), pp. 3215-3222.

[10] Schmidt D.P., Gopalakrishnan S., Jasak H., 2010, "Multi-dimensional simulation of thermal non-equilibrium channel flow", International Journal of Multiphase Flow, (36), pp. 284-292.

[11] Vallet A., Borghi R., 1999, C. R. Acad. Sci., Paris, Sér. II b, (327), pp. 1015-1020.

[12] Vallet A., Burluka A., Borghi R., 2001, "Development of an eulerian model for the "atomization" of a liquid jet", Atomisation and Sprays, (31), pp. 619-642.

[13] Downar-Zapolski, P., Bilicki, Z., Bolle, L., Franco, J., 1996, "The non-equilibrium relaxation model for onedimensional flashing liquid flow", International Journal of Multiphase Flow, (22), pp. 473-483.

[14] Menard T., Beau P. A., Tanguy S., Demoulin F. X., Berlemont A., 2005, "Primary break-up: DNS of liquid jet to improve atomization modelling", Computational Methods in Multiphase Flow III, (50), pp. 343-352.

[15] Lebas, R., Menard, T., Beau, P., Berlemont, A., Demoulin, F., 2009, "Numerical simulation of primary break-up and atomization: DNS and modelling study", International Journal of Multiphase Flow, (35), pp. 247-260.

[16] Pilch, M., Erdman, C.A., 1987, "Use of breakup time data and velocity history data to predict the maximum size of stable fragments for acceleration-induced break-up of a liquid drop", International Journal of Multiphase Flow, 13 (6), pp. 741-757.

[17] Weller H. G., Tabor G., Jasak H., Fureby C., 1998, "A tensorial approach to computational continuum mechanics using object-oriented techniques”, Comput. Phys., 12, pp. 620-631.

[18] Yildiz D., Van Beeck J., Rambaud P., Buchlin J. M., 17-20 July 2006, Proceedings of FEDSM2006, Joint U.S.European Fluids Engineering Summer Meeting.

[19] Yildiz D., 2005, Experimental investigation of superheated liquid jet atomization due to flashing phenomena, $\mathrm{PhD}$ thesis Université Libre de Bruxelles/von Karman Institute. ISBN: 978-2-930389- 49-4.

[20] Navarro-Martinez S., 2014, "Large eddy simulation of spray atomization with a probability density function method", International Journal of Multiphase Flow, 63, pp. 11-22.

[21] Schmidt, David P., 1997, Cavitation in Diesel Fuel Injector Nozzles. PhD thesis, Univ. of Wisconsin Madison. 